\title{
Role of the initial conditions on the enhancement of the escape time in static and fluctuating potentials
}

\author{
A.Fiasconaro *, D.Valenti, B.Spagnolo \\ INFM and Dipartimento di Fisica e Tecnologie Relative, Viale delle Scienze - \\ 90128 Palermo, Italy
}

\begin{abstract}
We present a study of the noise driven escape of an overdamped Brownian particle moving in a cubic potential profile with a metastable state. We analyze the role of the initial conditions of the particle on the enhancement of the average escape time as a function of the noise intensity for fixed and fluctuating potentials. We observe the noise enhanced stability effect for all the initial unstable states investigated. For a fixed potential we find a peculiar initial condition $x_{c}$ which separates the set of the initial unstable states in two regions: those which give rise to divergences from those which show nonmonotonic behavior of the average escape time. For fluctuating potential at this particular initial condition and for low noise intensity we find large fluctuations of the average escape time.
\end{abstract}

Key words: Statistical mechanics, Escape time, Noise enhanced stability, Metastable state

PACS: 05.40.-a,02.50.-r,05.10.Gg

\section{Introduction}

In the two last decades a remarkable increase has been done in the study of noise induced effects on nonlinear nonequilibrium systems, with attention also to complex and biological systems $[1,2,3,4]$. The introduction of noise and of a deterministic driving force in such a systems gives rise to counterintuitive effects which explain apparently anomalous behaviors in experiments. Nonlinear relaxation decay of physical systems from an initial unstable or metastable

\footnotetext{
* Corresponding author

Email address: afiasconaro@gip.dft.unipa.it (A.Fiasconaro).
} 
state involves fundamental aspects of non-equilibrium statistical mechanics. Examples of these resonance-like phenomena are stochastic resonance (SR) [3,5], resonant activation (RA) [6], noise enhanced stability (NES) [7,8], noise induced phase transitions (see ref.[1]), etc... A nonmonotonic behavior of the average escape time as a function of the noise intensity was revealed in a numerical study of a Brownian particle moving in a periodic fluctuating cubic potential [9]. This is the NES phenomenon: the stability of an otherwise unstable system can be enhanced by the presence of a finite amount of noise. The nonmonotonic behavior that often occurs in NES effect contradicts standard Kramers-like behavior, i.e. an exponential or monotonic decrease of the mean escape time with noise intensity [10]. The NES phenomenon was experimentally detected in the transient dynamics of an unstable physical system [7] and observed in different physical systems [11]. Recently some works have been done studying piecewise linear potentials with a metastable state, and exact evaluation of the decay time was obtained [8]. In this work we present a study of the average decay time of an overdamped Brownian particle subject to a cubic potential with a metastable state at different unstable initial conditions. A relevant result of this work is the existence, for fixed potential, of a peculiar initial position $x_{c}$ of the Brownian particle, which corresponds to the intersection point between the potential profile and the $x$-axis (see Fig. 1). For all the initial unstable states between the maximum of the potential and $x_{c}$, the average escape time diverges, while for initial positions $x_{o}>x_{c}$ we find a nonmonotonic behavior of the same quantity. Our results are consistent with that obtained in the case of piecewise linear potential profile [8]. For a periodic

fluctuating potential we recover the NES phenomenon for different values of the amplitude and of the frequency of the periodical driving force and large fluctuations of the average escape time as a function of the amplitude and the frequency at $x_{o}=x_{c}$.

\section{The Model}

The starting point of our study is the following Langevin equation

$$
\dot{x}=-\frac{\partial U(x, t)}{\partial x}+\sqrt{D} \xi(t)
$$

where $\xi(t)$ is the white Gaussian noise with the usual statistical properties: $\langle\xi(t)\rangle=0$ and $\langle\xi(t) \xi(t+\tau)\rangle=\delta(\tau)$, and

$$
U(x, t)=a x^{2}-b x^{3}-x A \cos (\omega t)
$$




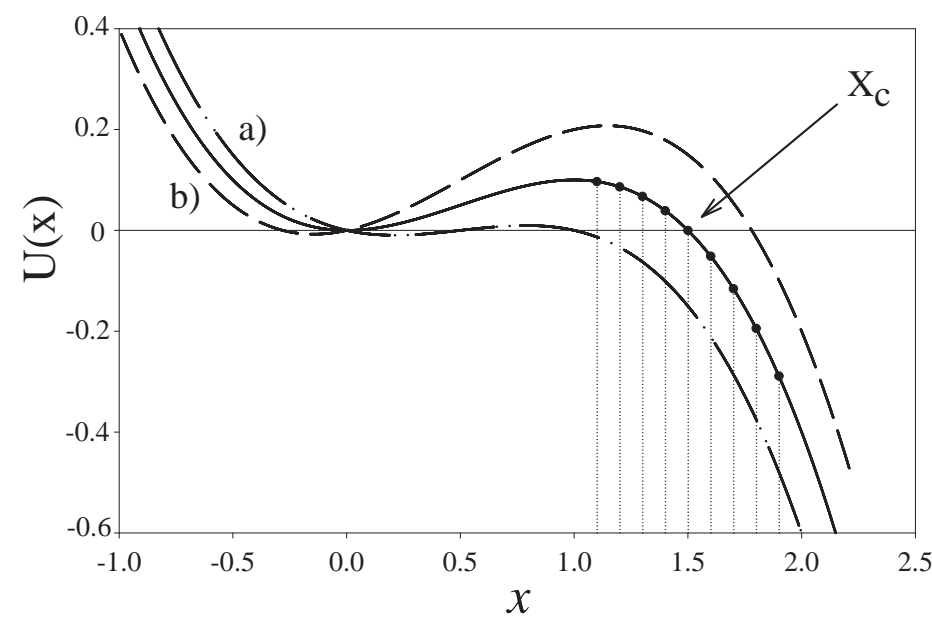

Fig. 1. The cubic potential $U(x)$ with the various initial positions investigated (dots); $x_{c}$ is the critical initial position. (a) and (b) indicate the limit curves of the oscillating potential. The absorbing boundary is $x_{F}=20$.

is the potential shown in Fig. 1, with $A$ and $\omega$ respectively the amplitude and the frequency of the driving force, and $a=0.3, b=0.2$. The potential profile has a local stable state at $x_{0}=0$ and an unstable state at $x_{0}=1$. For fixed potential $A=0$ the average escape time of a particle starting from $x_{o}$ and reaching a final position $x_{F}$ is given by [12]

$$
\tau\left(x_{0}, x_{F}\right)=\frac{2}{D} \int_{x_{0}}^{x_{F}} e^{2 u(z)} \int_{-\infty}^{z} e^{-2 u(y)} d y d z
$$

where $u(y)=\left(0.3 y^{2}-0.2 y^{3}\right) / D$ is a dimensionless potential profile. We evaluate this double integral partly analytically and partly numerically, splitting it as

$$
\tau\left(x_{0}, x_{F}\right)=\frac{2}{D} \int_{x_{0}}^{x_{F}} e^{2 u(z)} d z\left[\int_{-\infty}^{0} e^{-2 u(y)}+\int_{0}^{z} e^{-2 u(y)} d y\right]
$$

and evaluating analytically the first term inside the square parenthesis:

$$
\int_{-\infty}^{0} e^{-2 u(y)}=0.6046 e^{-z}\left[I_{-1 / 3}(z)+I_{1 / 3}(z)\right]-\frac{1}{2}{ }_{2} F_{2}\left(\frac{1}{2}, 1 ; \frac{2}{3}, \frac{4}{3} ;-2 z\right)
$$

where $z=1 /(10 D), I_{n}(z)$ is the modified Bessel function of the first kind and ${ }_{p} F_{q}\left(a_{1}, a_{2} ; b_{1}, b_{2} ; z\right)$ is the generalized hypergeometric function. 

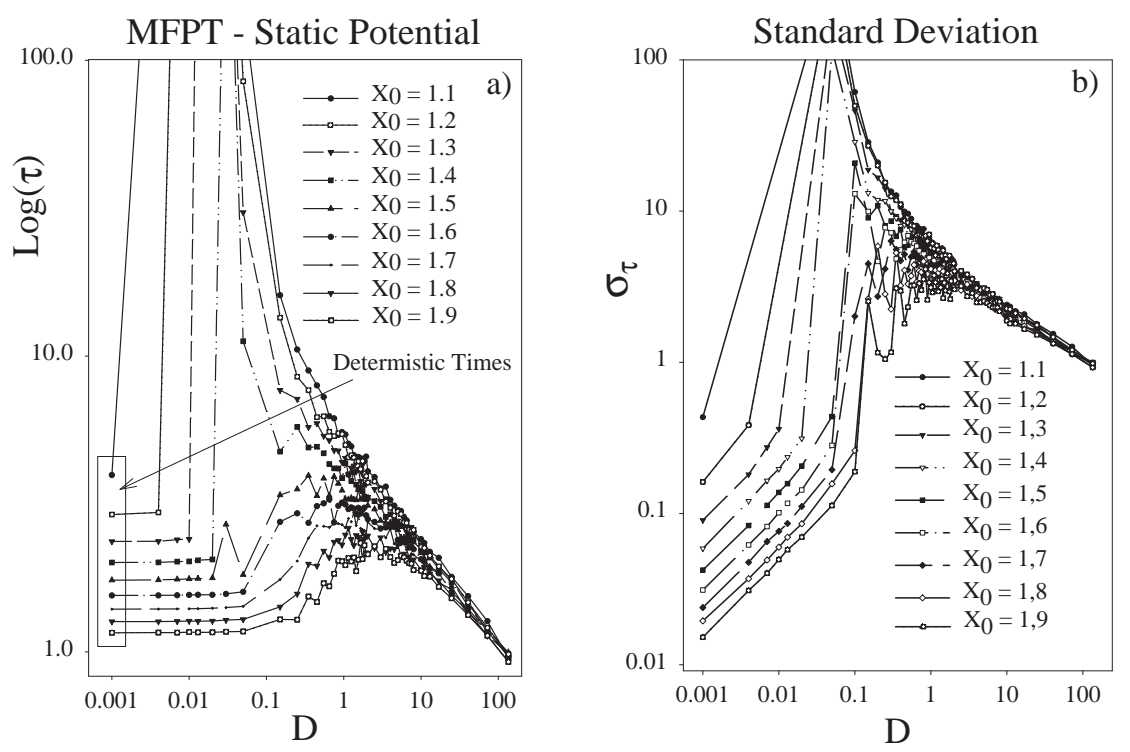

Fig. 2. a) Mean First Passage Time as a function of noise intensity for all the nine initial positions investigated (see Fig. 1 ), namely: $x_{o}=1.1 \div 1.9$, with steps of 0.1 . The number of realizations is 2000 and $x_{F}=20$. b) The standard deviation of the first passage time distribution, which shows a nonmonotonic behavior similar to that of MFPT.

\section{Results and Comments}

For all the initial unstable states beyond the potential barrier (see Fig. 1) we find an enhancement of the average escape time with respect to the deterministic time as a function of the noise intensity (Fig. 2). Specifically, for high values of the noise intensity with respect to the height of the barrier, we recover the Kramers behavior. For intermediate values of noise intensity, we observe an increase of the MFPT whose maximum goes to infinity when the initial position is in the range $x_{\max }<x_{o}<x_{c}$. These results are quite consistent with those obtained in the case of piecewise linear potential profiles [8], where a divergent behavior for $D \rightarrow 0$ is found. On the other hand for very low values of noise intensity we obtain the deterministic decay time. This effect, which appears in contradiction with the divergence for $D \rightarrow 0$, can be explained with the finite ensemble of particles in numerical experiments. Indeed, for low noise intensity the probability that some particle is trapped is very low and decreases exponentially to zero, that is only very few particles will be trapped. Only these particles, which represent very rare events for very small noise values, contribute to the enhancement of the escape time and give rise to the divergence. Therefore, because of this, we do not observe such particles in simulation and the average escape time becomes equal to the deterministic time. In Fig. 2 we can clearly see this effect. For initial positions approaching the maximum of the potential this effect is less pronounced, because the 
trapping probability of the particle is proportional to $\exp \left(-\Delta u\left(x_{o}\right) / D\right)$, where $\Delta u\left(x_{o}\right)$ is the potential barrier "seen" from the particle at $x(0)=x_{o}$. When the particle is near the maximum a relatively low amount of noise can push the particle back into the "stable" state, where it remains trapped for a long time, because of the low noise intensity. For higher values of initial position the amount of noise to put the particle into the potential well must be higher. This explains why the maximum of the average decay time is shifted towards higher values of the noise intensity. The observation time in digital simulation is finite, therefore the simulated escape time takes the maximum at this limiting point, and this is what we find (see Fig. 2a). For all the initial conditions within the range: $x_{m}<x_{o}<x_{c}$, we find that the average escape time can be more and more increased by the noise, while for initial conditions $x_{o}>x_{c}$ we obtain nonmonotonic behavior with a maximum and a finite average escape time when the noise intensity goes to zero. We calculate also the standard deviation $\left(\sigma_{\tau}\right)$ of MFPT as a function of the noise intensity for all the initial positions investigated (see Fig.2b). For $x_{o}>x_{c}$ we obtain a nonmonotonic behavior like the MFPT behavior. For $x_{o}<x_{c}$ the standard deviation grows towards divergent values for $D \rightarrow \infty$, and this corresponds to a big tail in the first passage time distribution. From the above analysis it appears that a peculiar initial position for the potential profile of Eq.(2) is $x_{o}=x_{c}=1.5$. We calculate then the theoretical average escape time from Eqs. $(4,5)$ for an initial position just after the cross point $x_{c}$ and we show the result in Fig. 3. The agreement between the numerical simulation of the SDE of the Eq. (1) and the theoretical evaluation is very good.

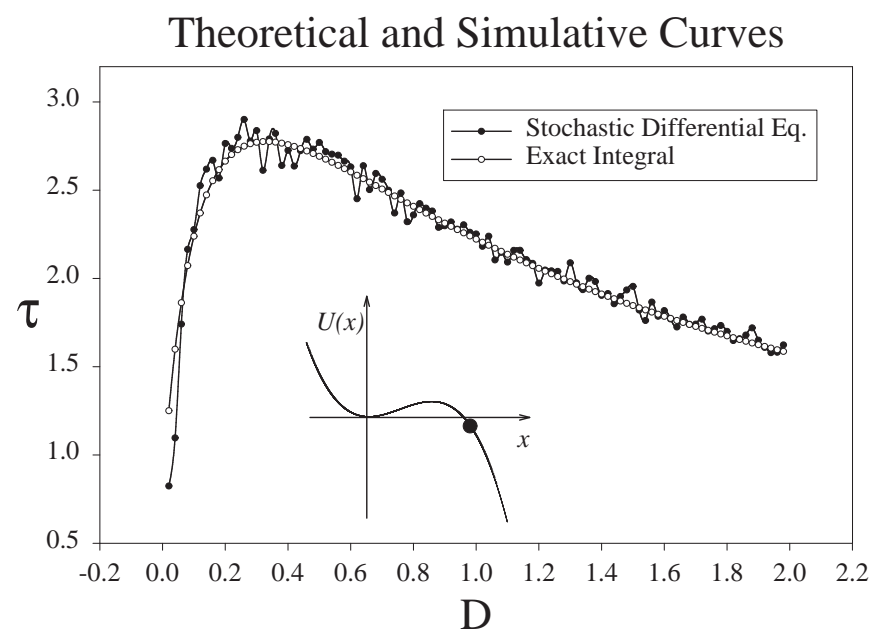

Fig. 3. Average escape time as a function of the noise intensity $D$, for an initial position $x_{o}=1.51>x_{c}=1.5$ and $x_{F}=2.2$. Comparison between theoretical MFPT (full circles) and the simulation of the SDE (empty circles)(Eq. (1)).

We calculate the average escape time at the static cross position $x_{c}=1.5$ for a periodical fluctuating cubic potential and the results are shown in Fig. 4 and 
Fig.5. Here we recover the enhancement of stability of the metastable state for all values of the amplitude and of the frequency of the periodical driving force investigated, with a nonmonotonic behavior of MFPT (a-figures). We find also that (b-figures): (i) the MFPT values are almost independent from the amplitudes and the frequencies of the driving force, except for the case $D=0.05$;

(ii) very large fluctuations of MFPT as a function of both the amplitude and the frequency of the periodic driving force at $D=0.05$ are present. The origin of these large fluctuations can be ascribed to the particular initial position represented by $x_{c}$, which is one of the two boundaries delimiting the parameter region $(\mathrm{A}, \nu)$ where NES effect can be observed [8]. Because of the oscillation of the potential between two limiting curves the intersection point is a function of the time $x_{c}(t)$ and as a consequence the Brownian particle experiences, during each period of the driving force, the two different dynamical regimes of the case of the static potential discussed above for $D \rightarrow 0$. Specifically the escape time passes from a divergent regime $\left(x_{c}(t)<x_{c}\right)$ to a convergent one $\left(x_{c}(t)>x_{c}\right)$, where $x_{c}$ is the intersection point for the static potential. This effect becomes more important for low noise intensity, i.e. for noise intensities less than the barrier height, as we can see in Figs. 4 and 5 for $D=0.05$. We obtain the same large fluctuations for other values of $D$ less than the barrier height (0.1), not reported here for sake of clearness of the figures. For values of amplitude and frequencies of the driving force higher then those presented in the figures, up to $\nu=20$ and up to $A=3$ respectively, the calculations confirm these large fluctuations. We also investigated the influence of different initial phases on the MFPT behavior as a function of the frequency and we recover large fluctuations again for $D=0.05$.

\section{Conclusions}

Nonmonotonic behavior of the mean escape time as a function of noise intensity is a noise-induced effect for nonlinear nonequilibrium systems with metastable states. In this work we analyzed the role of the initial conditions on the enhancement of the escape time from initial unstable states for a cubic potential. We obtain NES effect for static and periodical fluctuating potential and an enhancement of the NES effect for initial positions between the maximum of the potential well and the cross point $x_{c}$. We find also large fluctuations of the average escape time as a function of both the amplitude and the frequency of the sinusoidal force for low noise intensity, due to the different dynamical regimes experienced by the Brownian particle. Our results obtained for a particle moving in a cubic potential are quite general, because we always obtain NES effect when a particle is initially located just to the right of a local potential maximum, with a local minimum in its left side and the global escape region in its right side. 

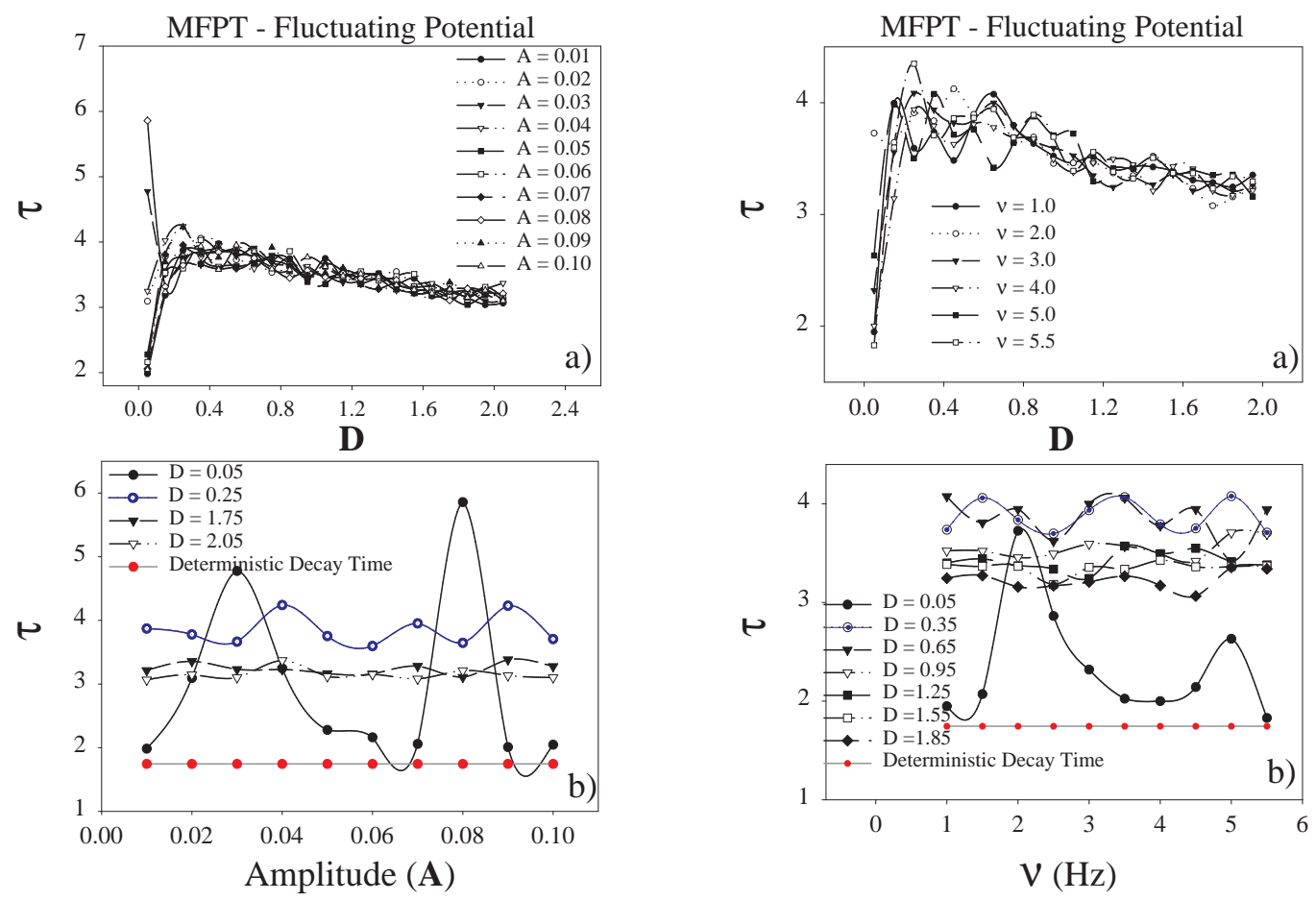

Fig. 4. MFPT as a function of noise intensity (a) and of the amplitude (b) of the driving force. The parameter values are: $x_{o}=1.5, \nu=10 \mathrm{~Hz}$.

Fig. 5. MFPT as a function of noise intensity (a) and of the frequency (b) of the driving force. The parameter values are: $x_{o}=1.5, A=0.02$.

\section{Acknowledgement}

We thank Dr. A. La Barbera for useful discussion and suggestions concerning the numerical simulations. This work was supported by INFM, MIUR and by INTAS Grant 01-0450.

\section{References}

[1] J. A. Freund, T. Pöschel (Eds.), Stochastic Processes in Physics, Chemistry, and Biology, Lecture Notes in Physics 557, Springer, Berlin, 2000.

[2] J. Parisi, S. Müller, W. Zimmermann (Eds.), Nonlinear Physics of Complex Systems, Springer, Berlin, 1996.

[3] L. Gammaitoni, P. Hänggi, P. Jung, F. Marchesoni, Rev. Mod. Phys. 70 (1998) 223 . 
[4] P. Talkner, P. Hänggi, New Trends in Kramers' Reaction Theory, Kluwer Academic Pub., 1995.

[5] R. N. Mantegna, B.Spagnolo, M. Trapanese, Phys. Rev. E 63 (2001) 011101.

[6] C.R. Doering, J.C. Gadoua, Phys. Rev. Lett. 69 (1992) 2318;

M.Marchi, F.Marchesoni, L.Gammaitoni, E.MenichellaSaetta, S. Santucci, Phys. Rew. E 54 (1996) 3479;

R.N.Mantegna, B.Spagnolo, Phys. Rev. Lett. 84 (2000) 3025.

[7] R.N.Mantegna, B.Spagnolo, Phys. Rev. Lett. 76 (1996) 563;

R.N.Mantegna, B.Spagnolo, Int. J. Bifurcation and Chaos 8 (1998) 783.

[8] N.V.Augdov, B.Spagnolo, Phys. Rev. E 64 (2001) 035102 R;

N.V.Augdov, B.Spagnolo, in Stochastic and Chaotic Dynamics in the Lakes: Stochaos, Ed. by D.S.Broomhead, E.A.Luchinskaya, P.V.E.McClintock, T.Mulin, AIP 502 (2000) 272.

[9] I.Dayan, M.Gitterman, G.H.Weiss, Phys. Rev. A 46 (1992) 757.

[10] H. A: Kramers, Physica 7 (1940) 284;

P.Hänggi, P.Talkner, M.Borkovec, Rev. Mod. Phys. 62 (1990) 251.

[11] J. E. Hirsch, B. A. Huberman, D. J. Scalapino, Phys. Rev. A 25 (1982) 519;

N. V. Agudov, A. N. Malakhov, Phys. Rev. E 60 (1999) 6333;

F. Apostolico, L. Gammaitoni, F.Marchesoni, S. Santucci, Phys. Rev. E 55 (1997) 36;

D. Dan, M. C.Mahato, A. M. Jayannavar, Phys. Rev. E 60 (1999) 6421;

R. Wackerbauer, Phys. Rev. E 59 (1999) 2872;

A. Mielke, Phys. Rev. Lett. 84 (2000) 818.

[12] C.W.Gardiner, Handbook of Stochastic Methods, 2nd edition, Springer-Verlag. 